\title{
Palivizumab Prophylaxis Against Respiratory Syncytial Virus Infection in Children with Immunocompromised Conditions or Down Syndrome: A Multicenter, Post-Marketing Surveillance in Japan
}

\author{
Tomoko Kashiwagi $^{1} \cdot$ Yukiko Okada $^{1} \cdot$ Ken Nomoto $^{1}$
}

Published online: 11 September 2017

(C) The Author(s) 2017. This article is an open access publication

\begin{abstract}
Objective The aim of this study was to assess the safety and effectiveness of palivizumab for the prevention of lower respiratory tract infection (LRI) caused by respiratory syncytial virus (RSV) in children with immunocompromised conditions or Down syndrome.

Methods In this multicenter, post-marketing surveillance study (December 2013 to December 2015), children aged $\leq 24$ months with immunocompromised conditions or Down syndrome (without hemodynamically significant congenital heart disease) receiving palivizumab immunoprophylaxis during two RSV seasons were observed until 30 days after the final palivizumab injection. Safety [adverse events (AEs), serious AEs (SAEs), adverse drug reactions (ADRs), serious ADRs (SADRs)] and effectiveness (frequency, incidence, and duration of hospitalization due to RSV infections) were assessed.

Results Of 304 patients receiving palivizumab, 167 (54.9\%) had immunocompromised conditions, and 138 (45.4\%) had Down syndrome; 260 (85.5\%) completed palivizumab immunoprophylaxis. The annual mean $( \pm s-$ tandard deviation) number of doses was $5.3( \pm 2.4)$ per season. Overall, 220 AEs occurred in 99 patients (32.6\%), including 89 SAEs in 53 patients $(17.4 \%)$. Of these, 33 AEs in 25 patients $(8.22 \%$ ) were considered ADRs, and 13 ADRs in 11 patients $(3.62 \%)$ were considered SADRs. In four patients, five SADRs (nephroblastoma and asthma in
\end{abstract}

Electronic supplementary material The online version of this article (doi:10.1007/s40272-017-0264-y) contains supplementary material, which is available to authorized users.

Ken Nomoto

ken.nomoto@abbvie.com

AbbVie GK, Mita 3-5-27, Minato-ku, Tokyo 108-6302, Japan the same patient, septic shock, device-related infection, and drug-induced liver injury) were previously unreported; however, none were considered drug-related. During the observation period, five RSV infections occurred and two patients required hospitalization.

Conclusion Palivizumab was generally safe and effective for the prevention of LRI caused by RSV in newborns, infants, and children with immunocompromised conditions or Down syndrome up to the age of 24 months.

\section{Key Points}

This is the first study of palivizumab use in children with immunocompromised conditions or Down syndrome conducted in a real-world setting.

Palivizumab was well tolerated and effective in preventing hospitalization due to severe RSV infection.

\section{Introduction}

Respiratory syncytial virus (RSV) is the primary cause of serious lower respiratory tract infections (LRI) in infants, and virtually all children become infected at least once by 24 months of age [1]. Currently, the available treatments for severe LRI caused by RSV are symptomatic therapies, such as supplemental oxygen and mechanical ventilation [2], making the management of severe RSV infections (which can be fatal for patients) challenging for healthcare providers [3]. Therefore, prevention of RSV infection in high-risk infants is critical. In the absence of a vaccine, the 
only effective method for preventing severe RSV infection is prophylaxis with palivizumab (Synagis ${ }^{\circledR}$, AbbVie GK, Tokyo, Japan) [4, 5]. Palivizumab is a humanized, antiRSV monoclonal antibody (IgG1), exhibiting potent neutralizing and fusion-inhibiting activity via selective binding to the fusion protein of RSV subtypes A and B [6].

Palivizumab is recommended for the prevention of serious LRI caused by RSV in high-risk children, such as premature infants or infants with hemodynamically significant congenital heart diseases (hs-CHD), in a number of countries, including Japan [7-10]. In addition to premature infants and children with hs-CHD, immunocompromised newborns, infants, and young children are also at risk for RSV-related LRI according to a 2011 nationwide survey in Japan [2]. Based on the findings of this national study, several medical societies in Japan requested approval of an additional indication for the use of palivizumab in children. Consequently, palivizumab was approved for immunoprophylactic use in children with immunocompromised conditions or Down syndrome in 2013. Outside of Japan, no regulatory agency has approved palivizumab for use in immunocompromised children.

Only 28 Japanese patients with immunocompromised conditions were enrolled in the previously conducted phase III clinical trial of palivizumab [7]; therefore, we conducted this post-marketing surveillance study to assess the safety and effectiveness of palivizumab in children with compromised immune systems and Down syndrome in real-world clinical settings.

\section{Methods}

This prospective, multicenter, observational, post-marketing surveillance was conducted at 64 clinical sites throughout Japan between December 19, 2013, and December 3, 2015, during the 2013-2014 and 2014-2015 RSV seasons (the typical epidemic RSV season in Japan is from late summer to the following spring). This study conformed to the Good Post-Marketing Study Practice (GPSP; Ministry of Health, Labour and Welfare ordinance). The study protocol was reviewed and approved in advance by the Pharmaceuticals and Medical Devices Agency of Japan; for this reason, no ethical review by individual facilities participating in the study was required. Informed consent is not required for post-marketing observational studies that are conducted under the GPSP in Japan. A written agreement was established between AbbVie and each participating institution. Data pertaining to the use of palivizumab in routine clinical settings without intervention were collected; however, no additional data that were specific to individual patients were included in this study. The study employed a consecutive enrollment method to reduce potential selection bias.

\subsection{Inclusion Criteria}

Newborns, infants, or young children aged 24 months and under with immunocompromised medical conditions were eligible for study participation. Newborns, infants, or young children aged 24 months and under who have Down syndrome, and children $\leq 24$ months of age without a current hs-CHD if they had experienced persistent respiratory symptoms or regular outpatient treatment due to a respiratory tract infection in previous RSV seasons were also eligible for the study.

\subsection{Exclusion Criteria}

Patients with contraindications to palivizumab listed in the approved Japanese product label, those with known hypersensitivity to ingredients of palivizumab, or those with known RSV infection before hospitalization were excluded from the safety analysis set. Patients who met a violation of protocol were also excluded from the safety analysis set. Patients who did not meet Japanese clinical criteria for the use of palivizumab in children with immunocompromised conditions or Down syndrome [11] were excluded from the effectiveness analysis set. Patients who had already been hospitalized at the start of palivizumab administration were also excluded from the effectiveness analysis set (Fig. 1).

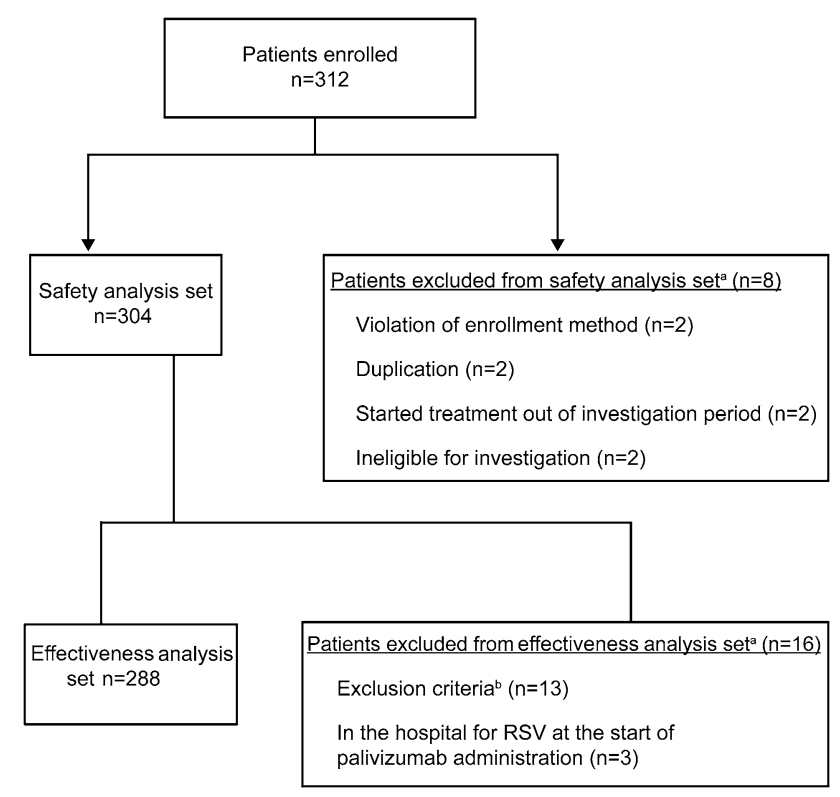

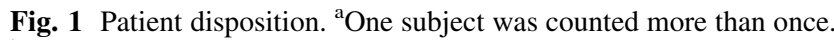
${ }^{\mathrm{b}}$ Exclusion criteria based on Mori et al. [9]. RSV respiratory syncytial virus 


\subsection{Dose Administration}

Palivizumab was administered by intramuscular injection at a dose of $15 \mathrm{mg} / \mathrm{kg}$ of body weight once each month throughout the RSV season.

\subsection{Study Procedures and Data Collection}

The observation period was defined as the interval from the first palivizumab injection to 30 days after the final injection, including instances of premature discontinuation. Observational visits were scheduled based on routine clinical practice, and prescription of palivizumab injection was performed by attending physicians. Patients who were hospitalized with symptoms lasting $\geq 48 \mathrm{~h}$ after admission with confirmed positive RSV laboratory tests were considered to be hospitalized due to RSV infection. However, if patients were hospitalized for RSV infection and had previously been hospitalized or discharged in the preceding 7 days, these occurrences were not recorded as hospitalizations due to RSV infection.

Data pertaining to patient characteristics, course of hospitalization, palivizumab administration, prior and concomitant drug use, adverse events (AEs), and RSV laboratory diagnostic tests including antigen, antibody (complement fixation test and neutralization test), culture, and polymerase chain reaction were collected.

\subsection{Safety Outcomes}

Safety was assessed as incidence of AEs, serious AEs (SAEs), adverse drug reactions (ADRs), and serious ADRs (SADRs). An AE was defined as any undesired medical occurrence, regardless of its cause. Causality of an $\mathrm{AE}$ in relation to palivizumab was classified into one of four categories: related, cannot rule out a possible relationship, indeterminable, or unrelated. Categorization of each $\mathrm{AE}$ was determined by attending physicians and AbbVie GK. When the attending physician, AbbVie GK, or both identified the $\mathrm{AE}$ as anything other than unrelated, the $\mathrm{AE}$ was considered an ADR. If an $\mathrm{AE}$ or ADR was determined by the investigator to cause death, a life-threatening condition, hospitalization or prolonged hospitalization, persistent or significant disability, congenital diseases or anomalies in the next generation, or other medically important conditions, it was classified as an SAE or as an SADR. AEs and ADRs were reported by system organ class (SOC) and by individual events.

\subsection{Effectiveness Outcomes}

Effectiveness was assessed as the rate of hospitalization due to RSV infections in patients who were receiving palivizumab for immunoprophylaxis.

\subsection{Sample Size}

In the prior phase III clinical trial of palivizumab in children with immunocompromised conditions and Down syndrome, the AEs of conjunctivitis (eye disorders) and encephalopathy (nervous system disorders) both had reported incidence rates of $3.6 \%(1 / 28)$, and were considered to have a possible or probable relationship to palivizumab [7]. The sample size of our current study was designed to detect at least one $\mathrm{AE}$ with an occurrence rate one-third the rate of AEs in the prior phase III trial (i.e., $1.2 \%$ ) [7], with over 95\% probability; therefore, 250 patients were required to participate in the study. The data set was to include 125 patients per year during the RSV season for 2 years (two seasons) to supplement the limited amount of data collected in the open-label clinical trial [7]. Assuming that $30 \%$ of the estimated number of patients who experienced each type of immunodeficiency (primary or secondary) were seen at participating medical institutions, the target number of patients was 14 per season $(28$ total) for primary immunodeficiency syndrome and 67 per season (134 total) for secondary immunodeficiency syndrome; 45 patients with Down syndrome were to be registered each season (90 total). The overall target number of patients was 252. Extension of the study was to be considered if one of the target numbers was not reached.

\subsection{Statistical Analysis}

The safety population consisted of all patients enrolled according to protocol with completed case report forms. Patients were not included if they were enrolled at a noncontracting institution or department (or if the institution had surpassed the contracted number of enrolled patients), had duplicate enrollment, did not receive palivizumab, or were administered palivizumab outside of the investigation period. The effectiveness population consisted of patients included in the safety analysis set who also met study eligibility criteria.

Continuous variables are presented as the number of non-missing values, mean [standard deviation (SD)], and median (minimum and maximum). Categorical variables are described by frequency and percentage.

\section{Results}

\subsection{Patients}

A total of 312 patients were enrolled from 64 hospitals in Japan that provide medical care for children with immunocompromised conditions or Down syndrome (Fig. 1). All clinical sites involved in this study were 
hospitals (no clinics were included), consisting of 35 university hospitals, two national hospitals, 16 local public hospitals, and 11 hospitals with unspecified designations. No limitations regarding the clinical specialty area were placed on attending physicians involved in this study. The safety population consisted of 304 patients; eight patients were excluded due to violations of enrollment methodology, duplication of records, initiation of treatment outside of the investigation period, or ineligibility for further investigation. The effectiveness population consisted of 288 patients; 13 patients did not meet inclusion criteria for effectiveness analysis, and three patients were hospitalized for RSV infection at the time of initial palivizumab administration. In all, $61.5 \%$ (187/304) of patients were male and $97.7 \%$ (297/304) were Japanese (Table 1). Mean $( \pm \mathrm{SD})$ age was $11.9( \pm 7.5)$ months, mean $( \pm \mathrm{SD})$ body weight was $7493.2 \mathrm{~g}( \pm 2486.3 \mathrm{~g})$, and mean $( \pm \mathrm{SD})$ gestational age and body weight at birth were $37.8( \pm 2.0)$ weeks and $2771.1 \mathrm{~g}( \pm 513.5 \mathrm{~g})$, respectively. Adrenocortical steroids, immunosuppressants, and antitumor drugs were the most commonly administered prior medications and concomitant drugs [Supplementary Table 1, see electronic supplementary material (ESM)].

\subsection{Palivizumab Administration}

Palivizumab was administered to 167 patients (54.9\%) with immunocompromised conditions and to 138 patients (45.4\%) with Down syndrome without hs-CHD; there was one patient with both an immunocompromised condition and Down syndrome without hs-CHD (Table 2). Myelosuppressive chemotherapy $(n=68)$, solid organ transplantation $(n=33)$, and hematopoietic stem cell allograft $(n=25)$ were the most common immunocompromised conditions for which palivizumab was administered. Mean $( \pm \mathrm{SD})$ number of palivizumab doses during each RSV season was $5.3( \pm 2.4)$ doses. Mean $( \pm \mathrm{SD})$ dose and dose interval were $14.87 \mathrm{mg} / \mathrm{kg}( \pm 1.08)$ and 31.4 days $( \pm 7.4)$, respectively. A total of $85.5 \%$ (260/304) of patients completed the planned course of palivizumab prophylaxis, while $14.5 \%$ (44/304) discontinued administration (Table 2). The most common reasons for palivizumab discontinuation were AEs [2.3\% (7/304)] and failure to appear for clinic visits or transfer to another hospital [8.2\% (25/304)].

\subsection{Safety}

A total of 220 AEs were reported in 99 patients (32.56\%), including 89 SAEs in 53 patients $(17.43 \%)$. The most commonly reported AEs and SAEs by SOC were infections and infestations [56 AEs (18.42\%) and 29 SAEs (9.54\%)] and respiratory, thoracic, and mediastinal disorders [36
Table 1 Baseline characteristics, $(N=304)$ (immunocompromised conditions, $n=167^{\mathrm{a}}$; Down syndrome, $n=138^{\mathrm{a}}$ )

\begin{tabular}{ll}
\hline Characteristics & $n(\%)^{\mathrm{b}}$ \\
\hline Gender & \\
Male & $187(61.5)$ \\
Female & $117(38.5)$ \\
Race & \\
Japanese & $297(97.7)$ \\
Asian, non-Japanese & $3(1.0)$ \\
Other & $4(1.3)$
\end{tabular}

Age in months at the start of palivizumab administration

$\begin{array}{ll}N & 304 \\ \text { Mean (SD) } & 11.9(7.5) \\ \text { Median (min-max) } & 11.5(0-24) \\ \text { Gestational age in weeks } & \\ N & 294 \\ \text { Mean (SD) } & 37.8(2.0) \\ \text { Median (min-max) } & 38.0(28-42) \\ \text { Presence of comorbidity } & \\ \text { Yes } & 183(60.2) \\ \text { Respiratory disease } & 50(16.4) \\ \text { Cardiovascular disease } & 45(14.8) \\ \text { Hepatic disease } & 21(6.9) \\ \text { Renal disease } & 18(5.9) \\ \text { Other } & 142(46.7) \\ \text { Unknown } & 1(0.3) \\ \text { Renal impairment } & \\ \text { Yes } & 32(10.5) \\ \text { Unknown } & 1(0.3) \\ \text { Hepatic impairment } & \\ \text { Yes } & 25(8.2) \\ \text { Unknown } & 1(0.3)\end{array}$

$S D$ standard deviation, min minimum, max maximum

a One subject was counted more than once

b unless otherwise specified

AEs (11.84\%) and 9 SAEs (2.96\%)]. The most frequently reported individual AEs were upper respiratory tract inflammation $[23(7.57 \%)]$, febrile neutropenia [11 (3.62\%)], and pneumonia [7 (2.30\%)]. The most frequently reported individual SAE was pneumonia [6 (1.97\%)] (Supplementary Table 2, see ESM).

A total of 33 ADRs were reported in 25 patients (8.22\%), including 13 SADRs in 11 patients (3.62\%) (Table 3). The most commonly reported ADRs and SADRs by SOC included infections and infestations [14 ADRs $(4.61 \%)$ and 8 SADRs (2.63\%)] and respiratory, thoracic and mediastinal disorders [6 ADRs (1.97\%) and 2 SADRs $(0.66 \%)]$. The most frequently reported individual ADRs were upper respiratory tract inflammation [5 (1.64\%)], pneumonia [3 (0.99\%)], and RSV infection [3 (0.99\%)], 
Table 2 Palivizumab administration, $(N=304)$

\begin{tabular}{|c|c|}
\hline & $n(\%)$ \\
\hline \multicolumn{2}{|l|}{ Criteria for prophylactic administration $^{\mathrm{a}}$} \\
\hline Immunocompromised conditions & $167(54.9)$ \\
\hline a. Myelosuppressive chemotherapy & $68(22.4)$ \\
\hline b. Solid organ transplantation & $33(10.9)$ \\
\hline c. Hematopoietic stem cell allograft & $25(8.2)$ \\
\hline d. Immunosuppressive therapy & $21(6.9)$ \\
\hline e. Others ${ }^{\mathrm{a}}$ & $17(5.6)$ \\
\hline On chronic peritoneal dialysis/hemodialysis & $16(5.3)$ \\
\hline Congenital nephrotic syndrome & $5(1.6)$ \\
\hline $\begin{array}{l}\text { f. Primary immunodeficiency syndrome with } \\
\text { abnormal T-cell function }\end{array}$ & $16(5.3)$ \\
\hline g. High-dose adrenocortical steroid therapy & $15(4.9)$ \\
\hline $\begin{array}{l}\text { h. Autologous hematopoietic stem cell } \\
\text { transplantation }\end{array}$ & $3(1.0)$ \\
\hline i. Bone marrow failure (e.g., aplastic anemia) & $3(1.0)$ \\
\hline j. Use of biologics & $2(0.7)$ \\
\hline k. Acquired T-cell dysfunction (e.g., AIDS) & $1(0.3)$ \\
\hline Down syndrome with no congenital heart disease & $138(45.4)$ \\
\hline 1. Marked megaloglossia/glossoptosis & $77(25.3)$ \\
\hline 2. Pulmonary hypertension & $29(9.5)$ \\
\hline 3. Airway obstruction due to respiratory tract malacia & $20(6.6)$ \\
\hline 4. Others ${ }^{\mathrm{b}}$ & $12(3.9)$ \\
\hline $\begin{array}{l}\text { 5. History of hospitalization due to viral infections/ } \\
\text { respiratory infections }\end{array}$ & $11(3.6)$ \\
\hline 6. Apnea & $4(1.3)$ \\
\hline 7. Pulmonary hypoplasia/dysplasia & $3(1.0)$ \\
\hline 8. Low lymphocyte or T-cell counts & $2(0.7)$ \\
\hline 9. Emphysematous lung & $1(0.3)$ \\
\hline \multicolumn{2}{|l|}{ Criteria for prophylactic administration (pooled) ${ }^{\mathrm{a}}$} \\
\hline Immunocompromised conditions & $167(54.9)$ \\
\hline $\begin{array}{l}\text { Hematopoietic malignancy, solid tumor, bone } \\
\text { marrow failure, hematopoietic stem cell } \\
\text { transplantation and solid organ transplantation }\end{array}$ & $126(41.4)$ \\
\hline $\begin{array}{l}\text { Kidney disease, rheumatism/inflammatory disease } \\
\text { and use of immunosuppressive drug }\end{array}$ & $46(15.1)$ \\
\hline Congenital/acquired immunodeficiency ${ }^{\mathrm{e}}$ & $17(5.6)$ \\
\hline Down syndrome & $138(45.4)$ \\
\hline \multicolumn{2}{|l|}{ Status of final administration } \\
\hline Completed & $260(85.5)$ \\
\hline Discontinued $^{\mathrm{a}}$ & $44(14.5)$ \\
\hline \multicolumn{2}{|l|}{ Reason for discontinuation } \\
\hline $\begin{array}{l}\text { Failed to appear for a clinic visit/transferred to } \\
\text { another hospital }\end{array}$ & $25(8.2)$ \\
\hline Others & $12(3.9)$ \\
\hline Adverse event & $7(2.3)$ \\
\hline
\end{tabular}

Table 2 continued

\begin{tabular}{lc}
\hline & $n(\%)$ \\
\hline Request of a family member & $1(0.3)$ \\
\hline$A I D S$ acquired immunodeficiency syndrome \\
${ }^{\text {a }}$ One subject was counted more than once \\
b The number of subjects with other findings only or unspecified \\
findings was 13 \\
${ }^{c}$ Based on criteria a, b, c, h, i for prophylactic administration [11] \\
d Based on criteria d, e, g, $\mathrm{j}$ for prophylactic administration [11] \\
e Based on criteria $\mathrm{f}$ and $\mathrm{k}$ for prophylactic administration [11]
\end{tabular}

and the most frequently reported individual SADR was pneumonia [3 $(0.99 \%)$ ].

In four patients, there were five SADRs that were previously unreported according to the existing product label in Japan: septic shock, nephroblastoma and asthma in the same patient, device-related infection, and drug-induced liver injury.

The first of these patients was a 23-month-old female born at 40 weeks gestational age who suffered fatal septic shock. Palivizumab was prescribed for congenital nephrotic syndrome with chronic peritoneal dialysis/hemodialysis. Palivizumab administration was discontinued as a result of death, and the relationship of the SADR to the study drug was categorized as indeterminable.

The second patient's SADRs were asthma and nephroblastoma. Palivizumab was prescribed for congenital nephrotic syndrome with chronic peritoneal dialysis/hemodialysis; the patient also had a history of Denys-Drash syndrome. Palivizumab administration was continued and nephroblastoma was identified after the last dose. This patient recovered from asthma and there was no change in the prognosis of nephroblastoma. For both SADRs, relationship to the study drug was categorized as indeterminable.

The third patient experienced a device-related infection. Palivizumab was prescribed for chronic peritoneal dialysis/ hemodialysis. This patient had numerous comorbid conditions including autosomal recessive polycystic kidney disease, chronic kidney disease, asthma, hypertension, and hypothyroidism. Palivizumab administration was continued and the patient recovered from the SADR. The relationship of the SADR to the study drug was categorized as indeterminable.

The fourth patient experienced drug-induced liver injury. Palivizumab was prescribed for significant bone marrow suppression caused by chemotherapy. This patient 
Table 3 Adverse drug reactions

Cumulative total in the specified use-results study $(N=304)$

Total Serious

\begin{tabular}{|c|c|c|}
\hline \multicolumn{3}{|l|}{ Adverse events } \\
\hline Number of subjects (events) & $99(220)$ & $53(89)$ \\
\hline Incidence $(\%)$ & 32.56 & 17.43 \\
\hline \multicolumn{3}{|l|}{ Adverse reactions } \\
\hline Number of subjects (events) & $25(33)$ & $11(13)$ \\
\hline Incidence $(\%)$ & 8.22 & 3.62 \\
\hline \multicolumn{3}{|l|}{ Type of adverse reaction } \\
\hline Infections and infestations & $14(4.61)$ & $8(2.63)$ \\
\hline Pneumonia & $3(0.99)$ & $3(0.99)$ \\
\hline Respiratory syncytial virus infection & $3(0.99)$ & $2(0.66)$ \\
\hline Influenza & $2(0.66)$ & \\
\hline Nasopharyngitis & $2(0.66)$ & \\
\hline Septic shock ${ }^{\mathrm{a}}$ & $1(0.33)$ & $1(0.33)$ \\
\hline Pneumonia, bacterial & $1(0.33)$ & $1(0.33)$ \\
\hline Acute sinusitis $^{\mathrm{a}}$ & $1(0.33)$ & \\
\hline Bronchitis & $1(0.33)$ & $1(0.33)$ \\
\hline Conjunctivitis $^{\mathrm{a}}$ & $1(0.33)$ & \\
\hline Gastroenteritis $^{\mathrm{a}}$ & $1(0.33)$ & \\
\hline Device-related infection $^{\mathrm{a}}$ & $1(0.33)$ & $1(0.33)$ \\
\hline Respiratory, thoracic, and mediastinal disorders & $6(1.97)$ & $2(0.66)$ \\
\hline Upper respiratory tract inflammation & $5(1.64)$ & $1(0.33)$ \\
\hline Asthma $^{\mathrm{a}}$ & $1(0.33)$ & $1(0.33)$ \\
\hline Gastrointestinal disorders & $2(0.66)$ & \\
\hline Diarrhea & $2(0.66)$ & \\
\hline Hepatobiliary disorders & $2(0.66)$ & $1(0.33)$ \\
\hline Liver disorder ${ }^{\mathrm{a}}$ & $1(0.33)$ & \\
\hline Drug-induced liver injury ${ }^{a}$ & $1(0.33)$ & $1(0.33)$ \\
\hline Neoplasms, benign, malignant, and unspecified (including cysts and polyps) & $1(0.33)$ & $1(0.33)$ \\
\hline Nephroblastoma $^{\mathrm{a}}$ & $1(0.33)$ & $1(0.33)$ \\
\hline Immune system disorders & $1(0.33)$ & \\
\hline Graft-versus-host disease ${ }^{a}$ & $1(0.33)$ & \\
\hline Endocrine disorders & $1(0.33)$ & \\
\hline Adrenal insufficiency ${ }^{a}$ & $1(0.33)$ & \\
\hline Metabolism and nutrition disorders & $1(0.33)$ & \\
\hline Hypokalemia $^{\mathrm{a}}$ & $1(0.33)$ & \\
\hline Vascular disorders & $1(0.33)$ & \\
\hline Hypertension $^{\mathrm{a}}$ & $1(0.33)$ & \\
\hline Renal and urinary disorders & $1(0.33)$ & \\
\hline Nephrotic syndrome $\mathrm{a}^{\mathrm{a}}$ & $1(0.33)$ & \\
\hline
\end{tabular}

Adverse reactions were classified using Medical Dictionary for Regulatory Activities/Japanese Version 18.1

${ }^{\text {a }}$ Adverse reactions/infections previously not mentioned in the precautions of the Japanese package insert for palivizumab

had numerous comorbid conditions (Pneumocystis pneumonia, bacterial pneumonia, sepsis, neutropenia, hypertension, hypogammaglobulinemia, atopic dermatitis, irondeficiency anemia, and constipation). Palivizumab administration was continued and the patient showed improvement in the SADR; relationship to the study drug was categorized as 'could not rule out a possible relationship.'

The remaining eight SADRs, which were included in the Japanese clinical guidelines for the use of palivizumab, 
consisted of four events of recovery and four events of remission.

\subsection{Effectiveness}

Of the 288 patients in the effectiveness analysis population, five patients $(1.7 \%)$ had an RSV infection during the investigation period (two RSV seasons), and two patients $(0.7 \%)$ were hospitalized. Both hospitalized patients were males with Down syndrome without hs-CHD; the first patient was 40 weeks' gestational age and was hospitalized for 8 days. The second patient was 37 weeks' gestational age, was hospitalized for 10 days, and required supplemental oxygen for 4 days.

\section{Discussion}

In this prospective, multicenter, post-marketing surveillance, we evaluated the safety and effectiveness of palivizumab in children with immunocompromised conditions and Down syndrome. Down syndrome patients with hsCHD were not included in this surveillance as palivizumab has been approved in Japan for children with hs-CHD [9]. Patients in the effectiveness analysis set received palivizumab according to existing Japanese clinical guidelines for use in this population [11]. These guidelines recommend the use of palivizumab in children with the following immunocompromised conditions: congenital or acquired immunodeficiencies; hematological malignancies, solid tumors, or bone marrow deficiencies; and treatment with immunosuppressive medications for kidney diseases, rheumatic/inflammatory diseases, hematopoietic stem-cell transplantation, or organ transplantation. For children with Down syndrome, the guidelines recommend palivizumab immunoprophylaxis for any of the following conditions: anatomical, physiological, or functional abnormalities of the respiratory system; history of hospitalization due to viral infection or respiratory infection; and abnormal immunological function by laboratory evaluation.

Palivizumab was well tolerated and effective in preventing severe RSV infection in real-world settings. The safety profile of palivizumab in this study was consistent with prior studies conducted in different high-risk populations, including premature infants (gestational age $\leq 35$ weeks), children with bronchopulmonary dysplasia, and children with hs-CHD [12-15].

The incidence of AEs, SAEs, ADRs, and SADRs was $32.6,17.4,8.2$, and $3.6 \%$, respectively. In each of these categories, infections and infestations were the most common type of event; this finding was not unexpected, considering that the study population consisted of children with compromised immune systems who were therefore already at risk for infection. Out of 13 SADRs, 11 resulted in recovery or remission. Discontinuation of palivizumab was mainly due to failure to appear for clinic visits or transfer to another hospital. Despite the occurrence of five previously unreported SADRs, none were determined to be causally related to palivizumab. Furthermore, the underlying indications for which palivizumab was administered, in addition to patient comorbidities, may have contributed to the development of the observed SADRs. In three of five previously unreported SADRs, patients improved or recovered, and palivizumab was continued in these patients with no safety concerns thereafter. These results indicate that palivizumab immunoprophylaxis can be safely used for the prevention of RSV infection in children with immunocompromised conditions or Down syndrome.

Palivizumab is currently the only approved agent for the prevention of serious LRI caused by RSV in children at high risk for RSV disease [5]. In the present study, the incidence of RSV infection was low $(1.7 \% ; n=5)$; of the five patients with RSV infections, two were hospitalized. In a prior multicenter, open-label, uncontrolled study among 28 immunocompromised Japanese children $\leq 24$ months of age who received prophylaxis with palivizumab, none were hospitalized due to RSV infection [7]. Additionally, in a prospective, observational study of children with Down syndrome conducted in Canada, palivizumab was associated with a 3.6-fold reduction in the incidence rate ratio for RSV-related hospitalization during first 2 years of life [16].

This multicenter, prospective, post-marketing surveillance was the first real-world study to evaluate the safety and effectiveness of palivizumab in children with immunocompromised conditions or Down syndrome in Japan. Our findings provide valuable evidence supporting the safety of palivizumab in a population at high risk for RSV infection. However, since this was an uncontrolled observational study, the complete safety profile of palivizumab (e.g., relative to placebo) cannot be determined. Further, the criteria used for determining whether AEs were causally related or unrelated to palivizumab may have varied among the participating physicians, potentially affecting the outcome of the safety analysis. Due to low incidence rates of immunocompromised conditions and Down syndrome, the population was too small to conduct a legitimate assessment of effectiveness, and results of the safety analyses from these two groups were combined.

\section{Conclusions}

This study demonstrated that palivizumab was generally safe and effective for the prevention of LRI caused by RSV in newborns, infants, and children with 
immunocompromised conditions or Down syndrome up to the age of 24 months.

Acknowledgements We thank Colleen Wegzyn of AbbVie for her support in the interpretation of the data as well as the development of the manuscript. AbbVie participated in the interpretation of data, review, and approval of the publication. Editorial support, in the form of medical writing, assembling tables, and creating high-resolution images based on authors' detailed directions, collating author comments, copyediting, fact checking, and referencing, was provided by Dr. Alina Gomes, MD, Dr. Annirudha Chillar, MD, PhD, and Maribeth Bogush, $\mathrm{PhD}$, of Cactus Communications, and was also funded by AbbVie GK.

\section{Compliance with Ethical Standards}

Funding This study was supported for funding and data collection by AbbVie GK, Tokyo, Japan.

Conflict of interest All authors are full-time employees of AbbVie GK.

Ethical approval This study conformed to the Good Post-marketing Study Practice (GPSP; MHLW ordinance). The study protocol was reviewed and approved in advance by the Pharmaceuticals and Medical Devices Agency, Japan. For this reason, no ethical review by the individual facilities participating in the study was conducted. Because informed consent is not required for post-marketing observational studies that are conducted under the GPSP in Japan, the present study did not solicit informed consent from the patients.

Open Access This article is distributed under the terms of the Creative Commons Attribution-NonCommercial 4.0 International License (http://creativecommons.org/licenses/by-nc/4.0/), which permits any noncommercial use, distribution, and reproduction in any medium, provided you give appropriate credit to the original author(s) and the source, provide a link to the Creative Commons license, and indicate if changes were made.

\section{References}

1. Glezen W, Taber LH, Frank AL, Kasel JA. Risk of primary infection and reinfection with respiratory syncytial virus. Am J Dis Child. 1986;140(6):543-6. doi:10.1001/archpedi.1986. 02140200053026.

2. Mori M, Kawashima H, Nakamura H, et al. Nationwide survey of severe respiratory syncytial virus infection in children who do not meet indications for palivizumab in Japan. J Infect Chemother. 2011;17(2):254-63. doi:10.1007/s10156-010-0121-1.

3. American Academy of Pediatrics. Respiratory syncytial virus. In: Pickering LK, Baker CJ, Long SS, McMillan JA, editors. Red book: 2006 report of the committee on infectious diseases. 27th ed. Elk Grove Village: American Academy of Pediatrics; 2006. p. $560-6$.

4. Eiland LS. Respiratory syncytial virus: diagnosis, treatment and prevention. J Pediatr Pharmacol Ther. 2009;14(2):75-85. doi:10. 5863/1551-6776-14.2.75.

5. Kusuda S, Takahashi N, Saitoh T, et al. Survey of pediatric ward hospitalization due to respiratory syncytial virus infection after the introduction of palivizumab to high-risk infants in Japan. Pediatr Int. 2011;53(3):368-73. doi:10.1111/j.1442-200X.2010. 03249.x.

6. Synagis (palivizumab). Full prescribing information. MedImmune, LLC. 2016. https://www.synagis.com/content/dam/ website-services/us/308-synagis-com/SPP/Pdf/synagis.pdf. Accessed 3 Oct 2016.

7. Mori M, Onodera M, Morimoto A, et al. Palivizumab use in Japanese infants and children with immunocompromised conditions. Pediatr Infect Dis J. 2014;33(11):1183-5. doi:10.1097/INF. 0000000000000392.

8. Bocchini JA, Bernstein HH, Bradley JS, et al. Policy statementModified recommendations for use of palivizumab for prevention of respiratory syncytial virus infections. Pediatrics. 2009;124(6):1694-701.

9. Nakazawa M, Saji T, Ichida F, et al. Guidelines for the use of palivizumab in infants and young children with congenital heart disease. Pediatr Int. 2006;48(2):190-3. doi:10.1111/j.1442-200X. 2006.02179.x.

10. Simoes EA. Immunoprophylaxis of respiratory syncytial virus: global experience. Respir Res. 2002;3(1):S26-33. doi:10.1186/ rr187.

11. Mori M, Morio T, Ito S, et al. Risks and prevention of severe RS virus infection among children with immunodeficiency and Down's syndrome. J Infect Chemother. 2014;20(8):455-9. doi:10.1016/j.jiac.2014.05.001.

12. IMpact-RSV Study Group. Palivizumab, a humanized respiratory syncytial virus monoclonal antibody, reduces hospitalization from respiratory syncytial virus infection in high-risk infants. Pediatrics. 1998;102(3):531-7.

13. Feltes TF, Sondheimer HM, Tulloh RM, et al. A randomized controlled trial of motavizumab versus palivizumab for the prophylaxis of serious respiratory syncytial virus disease in children with hemodynamically significant congenital heart disease. Pediatr Res. 2011;70(2):186-91. doi:10.1203/PDR.0b013e31822 0a553.

14. Lacaze-Masmonteil T, Seidenberg J, Mitchell I, et al. Evaluation of the safety of palivizumab in the second season of exposure in young children at risk for severe respiratory syncytial virus infection. Drug Saf. 2003;26(4):283-91.

15. Robbie GJ, Makari D, Harris B, Losonsky GA, Jafri HS. Randomized, double-blind study of the pharmacokinetics and safety of palivizumab liquid formulation compared with lyophilized formulation. Infect Dis Ther. 2014;3(2):203-14. doi:10.1007/ s40121-014-0042-x.

16. Yi $\mathrm{H}$, Lanctot $\mathrm{KL}$, Bont $\mathrm{L}$, et al. Respiratory syncytial virus prophylaxis in Down syndrome: a prospective cohort study. Pediatrics. 2014;133(6):1031-7. doi:10.1542/peds.2013-3916. 